\title{
Misbehaving in the Corona crisis: The role of anxiety and unfounded beliefs
}

\author{
Nikola Erceg ${ }^{1}$ (D) - Mitja Ružojčić ${ }^{1} \cdot$ Zvonimir Galić $^{1}$ \\ Published online: 12 September 2020 \\ (C) Springer Science+Business Media, LLC, part of Springer Nature 2020
}

\begin{abstract}
The aim of our study was to explore psychological determinants of COVID-19 responsible behavior. We focused on trait anxiety and worry about the corona crisis, knowledge and unfounded beliefs about coronavirus and thinking dispositions (cognitive reflection, actively open-minded thinking, faith in intuition and science curiosity) that should drive knowledge and beliefs. Additionally, we tested the effectiveness of a one-shot intervention based on the "consider counter-arguments" debiasing technique in changing COVID-19 unfounded beliefs. We used a convenience sample of 1439 participants who filled in the questionnaire on-line. Comparison of latent means showed that the "consider counter-arguments" intervention did not affect unfounded beliefs. Structural equation model, conducted on 962 participants with data on all variables, indicated that greater worry and weaker endorsement of COVID-19 unfounded beliefs lead to more responsible COVID-19 behavior. The relationship of trait anxiety and thinking dispositions with the criterion was mediated through the worry about COVID-19 and unfounded beliefs about COVID-19, respectively.
\end{abstract}

Keywords Coronavirus $\cdot$ Responsible behavior $\cdot$ Unfounded beliefs $\cdot$ Actively open-minded thinking $\cdot$ Anxiety

\section{Introduction}

The goal of this research was to investigate psychological determinants of responsible behavior in the COVID-19 (C-19) crisis. We focused on two groups of possible antecedents. The first group are feelings of worry and anxiety that accompany the perception of the situation. The second group encompasses knowledge about the situation but also unfounded beliefs that frequently thrive in crises and, thus, possibly drive irresponsible behaviors. Finally, we also tested a "oneshot" intervention, namely a manipulation of instruction, aimed at improving formation of beliefs related to C-19 crisis.

A recent study showed that being exposed to C-19 information increases anxiety and worry (Sorokowski et al. 2020) and several strains of evidence show that worry and anxiety can have a positive effect on health behavior. First, both worry and

Electronic supplementary material The online version of this article (https://doi.org/10.1007/s12144-020-01040-4) contains supplementary material, which is available to authorized users.

Nikola Erceg

nerceg@ffzg.hr

1 Department of Psychology, Faculty of Humanities and Social Sciences, University of Zagreb, Ivana Lucica 3, 10 000 Zagreb, Croatia anxiety are generally related to responsible and protective health behavior. Recent reviews show that worry predicts adaptive preparation and planning, uptake of healthpromoting behaviors and seeking information in response to stressful events (Sweeny and Dooley 2017), but also increased uptake of tests to detect presence or risk of various cancers, intentions to obtain a Lyme disease vaccine or engage in safe sex behavior (McCaul and Mullens 2003). Second strain of evidence comes from the studies showing a positive relationship between worry and anxiety with risk aversion (see Hartley and Phelps 2012 for an overview). A final piece of evidence that anxiety and worry could be related with responsible behavior is related to the studies showing greater trust in authorities following a fear evoking events (Burke et al. 2013). As people are motivated to see the world as a secure and predictable place, in times of threat they can be more prone to affiliating themselves with cultural institutions that offer an actual or symbolic sense of security and safety (Lambert et al. 2011). In sum, given its relationship with responsible and protective behavior, risk-avoidance and trust in authorities, we expect to find a positive association between worry and responsible C19 related behavior, and believe that greater worry about the situation partially stems from the personality trait of anxiety (Spielberger 2010).

The research also show that a period of crisis and high uncertainty is especially fruitful for the emergence of 
conspiracy theories (Van Prooijen and Douglas 2017, 2018). In the times when people feel vulnerable, scared and helpless, conspiracy theories are attractive because they help to make sense of the situation by providing the answers about the emergence of the crisis and actors behind it (Van Prooijen and Douglas 2017). Thus, it is no wonder that misinformation related to C-19 disease proliferated in such a degree to deserve its own Wikipedia page (https://en.wikipedia.org/wiki/ Misinformation_related_to_the_2019\%E2\%80\%9320 coronavirus pandemic).

The conspiracy theories can seem absurd, but they present a danger if many people start believing in them and acting accordingly. One of the characteristics of conspiracy beliefs is that they are consequential: they impact behavior in important domains of life such as health, interpersonal relationships or safety (Van Prooijen and Douglas 2018). If a person believes that coronavirus is a hoax, (s)he is more likely to behave irresponsibly and spread the virus.

It seems that conspiracy beliefs are underpinned by a stable characteristic called conspiracy mentality (Imhoff and Bruder 2014) that explains why same people hold conspiracy and other unfounded beliefs across various domains (Ståhl and Van Prooijen 2018; Van Prooijen and Douglas 2018). So, the traits that were shown to underpin other unfounded beliefs could also lead to C-19 conspiracy beliefs. In our research we focused on cognitive reflection, faith in intuition, actively open-minded thinking and science curiosity.

Cognitive reflection refers to ability and/or disposition to engage in reflective thinking instead of relying on intuition and reporting a first response that comes to mind (Frederick 2005; Pennycook et al. 2016). Multiple findings consistently showed that it is a robust negative predictor of unfounded beliefs. Namely, cognitive reflection was predictive of lower endorsement of paranormal, superstitious, pseudoscientific and conspiracy beliefs (Pennycook et al. 2012; Pennycook et al. in press, 2015; Ståhl and Van Prooijen 2018; Šrol 2020) and higher endorsement of scientifically founded beliefs (McPhetres and Pennycook 2019; Pennycook et al. in press). Several recent studies (e.g. Pennycook et al. 2020; Teovanovic et al. 2020) found that these findings also extend to beliefs about C-19. Specifically, in these studies cognitive reflection was negatively related with belief in misinformation about C-19.

Particularly important for the formation of evidence-based beliefs is openness to new information and evidence that counteract current beliefs, leading to willingness to revise beliefs if new evidence deems it necessary. This disposition is called actively open-minded thinking (Baron 2018; Baron 2019; Baron et al. 2015). Svedholm-Häkkinen and Lindeman (2018) provide an overview of studies showing that actively open-minded thinking is a basis of rational thought. Actively open-minded thinking generally predicts lower endorsement of unfounded beliefs such as paranormal, conspiracy, supernatural, theistic and anti-science beliefs (Baron et al. 2015; Pennycook et al. 2014; Svedholm and Lindeman 2013; Svedholm-Häkkinen and Lindeman 2018; Swami et al. 2014; Pennycook et al. in press), but higher endorsement of pro-science claims (McPhetres and Pennycook 2019). Similarly to actively open-minded thinking, science curiosity, a disposition to seek out and consume scientific information for personal pleasure, was shown to promote open-minded engagement with evidence even when this evidence is contrary to individual's predispositions (Kahan et al. 2017).

Conversely, a disposition to trust one's initial hunches (i.e., faith in intuition) has been shown to predict higher endorsement of unfounded beliefs. Faith in intuition was positively correlated with different conspiracy beliefs (Swami et al. 2014) as well as other unfounded beliefs such as paranormal beliefs (Aarnio and Lindeman 2005) and superstitious thinking (Erceg et al. 2019; Sadler-Smith 2011). In sum, we expected cognitive reflection test, actively open-minded thinking and science curiosity to be negatively, and faith in intuition to be positively related to $\mathrm{C}-19$ related unfounded beliefs and, in turn, irresponsible behavior.

Earlier studies showed that actively open-minded thinking is a thinking style that can be practiced and learned (Baron et al. 2017). Thus, the goal of many debiasing techniques is to provoke actively open-minded thinking. One specific debiasing technique that has been shown to improve the quality of decisions is so called "consider-the-opposite" that nudges a decision-maker into generating rival points of view or imagining counterfactual outcomes for an event (Lilienfeld et al. 2009; Morewedge et al. 2015). This technique could also be particularly appropriate in nudging people towards more critical stance when forming beliefs. In recent review, van Prooijen (2019) concluded that the evidence overwhelmingly supports the Gullible Conspiracism Hypothesis by which the decision to reject official accounts of important events and subsequently embrace conspiracy theories is often made reflexively instead of reflectively. Not being based on rational arguments, once accepted, these beliefs are highly resilient to change. According to Van Prooijen (2019), confirmation bias and motivated reasoning play a crucial role here: believers selectively attend to and embrace evidence that support their suspicions, while rejecting evidence that disconfirm them. This results in an extensive theory that appears to be well elaborated and supported by a lot of evidence. Therefore, even asking people to consider that their beliefs might be wrong and to think about some arguments in favor of the opposite position might be beneficial in helping them form more accurate/scientifically backed beliefs. In line with this, a second goal of our study was to see whether the one-shot intervention based on considering counter-arguments debiasing technique could nudge people to revise their C-19 related beliefs. We did this by manipulating the instruction at the 
beginning of our survey. We gave a random half of the sample (a "control" group) a "usual" instruction to be thorough and not to skip questions as each of their responses matter to us, while the other random half ("experimental" group) received a "consider counter-arguments" instruction. Specifically, they were given the following information:

"Psychological research clearly shows that people make much more accurate judgments and decisions if, before reaching a conclusion, they think further about why their beliefs might be wrong and try to find arguments contrary to their current beliefs. Thus, psychological research shows that, in order to make better judgments and decisions, it is important:

a) Not to make judgments and come up with conclusions quickly and hastily.

b) To think about why your current belief might be wrong and try to find a few arguments against it.

This information can serve you in everyday life, but also when solving this survey."

\section{Methodology}

\section{Procedure}

We collected our sample in Croatia between 21st and 29th March 2020 through an on-line survey. We shared a link to the survey questionnaire on our department's Facebook page and on our personal pages on different social networks. We also posted the link in three Facebook groups concerning coronavirus news and sent the link privately to our friends and colleagues.
All participants answered the same set of questions presented in the same order to all participants, but the introduction to the questionnaire differed randomly among participants. For approximately half $(680,47.3 \%)$ of the sample the introduction included "consider counter-arguments" debiasing message. The debiasing message informed participants that psychological research indicate that people make more accurate conclusions if they don't make their judgment quickly but rather try to find the opposing arguments to their current beliefs before reaching final conclusion.

\section{Participants}

We used a convenience sample of 1439 participants, of which $996(69.2 \%)$ reached the end of the questionnaire and reported on their sociodemographic characteristics. Of these participants, $74.1 \%$ were females and their average age was 34.8 years $(S D=10.67, \min =15, \max =74)$. Most of the participants had a college or university degree $(79.8 \%)$ while $20.1 \%$ had a high school diploma.

All 1439 participants completed the items that asked about unfounded beliefs regarding C-19, given that they were at the beginning of the questionnaire. Therefore, we tested the effect of "consider counter-arguments" intervention on the full sample in order to increase the study's statistical power. We conducted the rest of the analyses on participants that had data on all the used variables, and we report sample sizes for each analysis in tables in the Result section.

\section{Instruments}

We report all the items we used in the supplemental online materials available at https://osf.io/9ht5k/ and omega coefficients of reliability (Raykov 2001) for the focal latent variables in Table 1.

Table 1 Descriptive statistics of focal variables

\begin{tabular}{|c|c|c|c|c|c|c|c|}
\hline & $n$ & $M$ & Lower CI & Upper CI & Minimum & Maximum & $\omega$ reliability \\
\hline Trait Anxiety & 1012 & 4.18 & 4.09 & 4.26 & 1.00 & 7.00 & .84 \\
\hline Cognitive Reflection & 1439 & 0.39 & 0.37 & 0.41 & 0.00 & 1.00 & .65 \\
\hline AOT & 1046 & 4.39 & 4.36 & 4.42 & 2.63 & 5.00 & .65 \\
\hline Faith in Intuition & 1043 & 3.42 & 3.38 & 3.47 & 1.00 & 5.00 & .84 \\
\hline Science Curiosity & 1001 & 0.00 & -0.05 & 0.04 & -2.39 & 1.35 & .73 \\
\hline COVID-19 Worry & 1437 & 5.26 & 5.19 & 5.33 & 1.00 & 7.00 & - \\
\hline COVID-19 Conspiracy Beliefs & 1439 & 2.30 & 2.25 & 2.36 & 1.00 & 6.00 & .86 \\
\hline COVID-19 Treatment Beliefs & 1383 & 2.00 & 1.95 & 2.04 & 1.00 & 5.17 & .83 \\
\hline COVID-19 Averaged Unfounded Beliefs & 1439 & 2.16 & 2.12 & 2.21 & 1.00 & 5.63 & .92 \\
\hline COVID-19 Knowledge & 1346 & 0.77 & 0.76 & 0.78 & 0.00 & 1.00 & .38 \\
\hline COVID-19 Behavior & 1323 & 4.39 & 4.36 & 4.42 & 1.00 & 5.00 & .57 \\
\hline
\end{tabular}

AOT Active Open-minded Thinking, CI Confidence Interval 
COVID-19 Responsible Behavior We used four items that asked participants to estimate how often, in the last 10 days, they performed each of the four desired behaviors regarding C-19: avoiding physical contact, washing hands, avoiding going out, and coughing and sneezing in elbow. We used a five-point scale where $1=$ never and $5=$ all the time and a total score was calculated as a mean of responses on these four behavior items. The reason we focused on these four behaviors was that, at the time the data collection (March, 2020), the four behaviors were the only publicly announced and officially recommended means of coping with coronavirus spread in the population. Some other behaviors that were latter shown to be even more efficient in this respect, such as hygienic facemasks, received very little attention at that time or its use was even actively discouraged by the officials (probably due to the general lack of protective hygienic equipment in the country). Importantly, the four recommended behaviors were not legally mandated so it was up to each individual to decide whether or not and to what degree they will follow them.

COVID-19 Related Worry We measured worry with one item that asked participants to assess the degree of their worry for themselves and their family on a scale from $1=$ not worried at all to $7=$ extremely worried.

Unfounded Beliefs about COVID-19 We captured two types of unfounded beliefs. Beliefs about conspiracies regarding coronavirus ( 8 items, e.g. "The coronavirus was created to reduce the world population") and beliefs about possible treatments of C-19 that are not scientifically backed up (6 items, e.g. "Colloidal silver is a potentially good cure for this coronavirus strain."). For both sets of items participants reported on their agreement on a six-point scale $(1=$ completely disagree; $6=$ completely agree). Total score was calculated as a mean score of the responses.

Given that we were interested in the relationships with unfounded beliefs about C-19 in general, we specified a measurement model in which conspiracy regarding coronavirus and beliefs about possible treatments latent factors loaded on a higher order unfounded beliefs factor. The model had an acceptable fit to the data $\left(\chi^{2}(76)=982.08, p<.001 ; \mathrm{CFI}=\right.$ 0.90; $\mathrm{RMSEA}=0.09,90 \% \mathrm{CI}[0.09,0.10], \mathrm{AIC}=55,851.329$ ) and the first order factors had high standardized loadings with the second order unfounded beliefs factor ( 0.58 for conspiracy factor and 0.87 for treatment factor). Therefore, we used the higher order unfounded beliefs factor in our analyses.

Knowledge about COVID-19 We measured knowledge using six objective knowledge C-19 questions (e.g. "Coronavirus mortality is higher among the elderly population") where participants responded with yes/no answer. Each correct response was awarded one point and the total score was a mean of correct responses on six questions.
Trait Anxiety We measured trait anxiety with four items from Johnson (2014). Example item is "I get stressed out easily". Participants estimated the degree to which each of the emotions described in the item applies to themselves in general using a seven-point scale ( $1=$ it does not apply to me at all; $7=$ it completely applies to me) and the total score was mean of these responses.

Cognitive Reflection We measured cognitive reflection with three Cognitive Reflection Test (CRT) items taken from currently existing versions of the CRT (Erceg et al. 2020) which we revised so that their content appears to be related to C-19. We coded each of the responses as correct/incorrect and the total score was a mean of correct responses on the three items.

Actively Open-Minded Thinking We measured actively openminded thinking with ten items from Baron (2019). Example item is "People should revise their conclusions in response to relevant new information". Participants assessed their agreement with items on a five-point scale ( $1=$ completely disagree; $5=$ completely agree) and the total score was mean of these responses. Due to two items being practically unrelated to others (number 3 and 9 in the supplemental online material), we discarded them from the analyses and proceeded with eight-item version.

Faith in Intuition We measured faith in intuition with a fiveitem scale (Norris et al. 1998). Example item is "My initial impressions of people are almost always right". The participants assessed how well each of the items describes themselves on a five-point scale ( $1=$ not at all; $5=$ very much). The total score was mean of these responses.

Science Curiosity We measured science curiosity with five items taken from Kahan et al. (2017). We asked participants to estimate how closely they follow science related news, read science related books, talk about science related topics, share science related posts on social networks and generally how interested they are in science. Given the different rating scales among the items, we calculated the total score on the scale by first standardizing the scores on each item and then averaging the standardized scores across items. We also included filler items related to other topics such as politics, sports and entertainment to make appear that a questionnaire is about general participants' interests and not only those related to science, but did not score these.

\section{Results}

The database we used as well as R-code for all the analyses are available at https://osf.io/9ht5k/. 
Test of the Consider Counter-Arguments Intervention

Prior to testing the prediction of $\mathrm{C}-19$ responsible behavior, we tested if our one-shot intervention based on the "consider counter-arguments" technique reduced unfounded beliefs of our participants. We compared latent means of unfounded belief higher order latent factor of the group that was exposed vs the group that was not exposed to the intervention. In both models we first equalized the loadings and the intercepts across the two groups, which is a precondition for the test of latent mean differences (Putnick and Bornstein 2016). Both the model in which loadings and intercepts were constrained but latent means differed among groups $\left(\chi^{2}(175)=1115.63\right.$, $p<.001 ; \mathrm{CFI}=0.90 ;$ RMSEA $=0.09,90 \%$ CI $[0.08,0.09]$, $\mathrm{AIC}=55,894.21)$ and the "constrained latent means model" showed similar fit to the data $\left(\chi^{2}(178)=1116.18, p<.001\right.$; $\mathrm{CFI}=0.90 ;$ RMSEA $=0.09,90 \% \mathrm{CI}[0.08,0.09], \mathrm{AIC}=$ $\left.55,888.76 ; \Delta \chi^{2}(3)=0.55, p=0.908\right)$. This meant that the "consider counter-arguments" intervention did not affect unfounded beliefs of our participants. Accordingly, we conducted the rest of our analyses only on full sample which included both participants that were exposed and not exposed to the intervention.

\section{Descriptive Statistics and Correlation Analyses}

Descriptive statistics and omega coefficients of reliability (Raykov 2001) for the focal latent variables are shown in Table 1. Intercorrelations among variables are shown in Table 2. We show both correlations among the total scale scores, calculated as mean of scores on the items, and correlations among latent factor scores, calculated through an "unrestricted" structural equation model estimated through maximum likelihood (ML) procedure. ${ }^{1}$ Given that we tested our hypotheses through SEM using latent factor scores and because these scores are corrected for unreliability, we comment only on the correlations among latent factors.

As can be seen, trait anxiety correlated with more worry on C-19 and higher amount of responsible C-19 behavior. Also, anxiety had a small negative correlation with C-19-related knowledge.

Cognitive reflection and thinking dispositions correlated in expected direction with C-19-related unfounded beliefs and knowledge. Cognitive reflection, actively open-minded thinking and science curiosity were related with less unfounded beliefs and better knowledge on C-19, while faith in intuition related with more unfounded beliefs and worse knowledge on C-19. As for the behavior, only actively open-minded thinking and science curiosity showed significant relation with more responsible behavior regarding $\mathrm{C}-19$ threat.

\footnotetext{
1 "Unrestricted" SEM model is a measurement model in which all latent factors are allowed to correlate with each other.
}

C-19 worry was related with more responsible behavior, while unfounded beliefs related with less responsible behavior regarding C-19. Knowledge on C-19 was not related to C-19 behavior.

We also correlated focal variables with several sociodemographic variables, namely: sex, age, education level and political values (liberal vs conservative). These variables had mostly lower correlations with C-19 worry, unfounded beliefs, knowledge and behavior. However, there were two noticeable correlations: male participants behaved less responsibly and conservative participants had more unfounded beliefs.

\section{The Predictors of COVID-19 Responsible Behavior}

To test the prediction of responsible behavior regarding C-19 threat, we estimated a structural equation model using ML estimator and confidence intervals $(\mathrm{CI})$ of parameter estimates calculated through 5000 bootstrap samples. We deemed the effect significant if the $95 \%$ bootstraped CI did not include zero. In the model, individual differences: anxiety, cognitive reflection, actively open-minded thinking, faith in intuition and science curiosity, served as predictors of C-19-related worry, unfounded beliefs, knowledge and responsible behavior, whereas C-19-related worry, unfounded beliefs and knowledge served as mediators of the effects of individual differences on the $\mathrm{C}-19$ responsible behavior. We note that C-19 related worry was measured with one item and it is therefore included in the model as an observed variable. Knowledge on C-19 was measured with six loosely related items about various aspects of coronavirus and C-19 so we believed it was more appropriate to include it as an index calculated as mean of correct responses on the six questions rather than a latent variable. Rest of the variables in the regression portion of the model were latent. We calculated indirect effects by multiplying the effect of a predictor on a mediator with the effect of a mediator on the criterion while controlling for the effect of the predictor on the criterion. The tested model showed an appropriate fit to the data $\left(\chi^{2}(910)=2575.77\right.$, $p<.001 ; \mathrm{CFI}=0.88$; RMSEA $=0.04,90 \%$ CI $[0.04,0.05]$, $\mathrm{AIC}=107,018.634)$. We show the structural/regression portion of the model with only significant parameter estimates in Fig. 1, while all regression estimates are shown in the online supplemental materials. ${ }^{2}$

In total, we explained $11.4 \%$ variance of C-19-related worry. As for the "emotional part" of our model, trait anxiety predicted how concerned are the participants about the situation and that variable predicted $\mathrm{C}-19$ responsible behavior. Neither of the "cognitive" variables was related to COVID-

\footnotetext{
${ }^{2}$ We also ran a model in which we included sociodemographic variables as controls and the results were largely the same. The regression estimates from model with controls are also available in the online supplemental materials.
} 
Table 2 Intercorrelations among mean scores (above diagonal; $n=998$ ) and latent factor scores (below diagonal; $n=942$ ) of focal and sociodemographic variables

\begin{tabular}{|c|c|c|c|c|c|c|c|c|c|c|c|c|c|c|}
\hline & $M(S D)$ & 1. & 2. & 3. & 4. & 5. & 6. & 7. & 8. & 9. & 10. & 11. & 12. & 13. \\
\hline 1. Sex $(1=$ female, $2=$ male $)$ & $1.26(0.44)$ & & .03 & -.06 & $.13^{* *}$ & $-.18^{* *}$ & $.18^{* *}$ & $.11^{* *}$ & -.06 & .04 & $-.11 * *$ & $-.10 * *$ & $.15^{* *}$ & $-.21 * *$ \\
\hline 2. Age & $34.80(10.67)$ & .03 & & $.19 * *$ & $-.07 *$ & $-.08 *$ & $-.12 * *$ & .02 & .03 & .00 & $.18 * *$ & .05 & .00 & $.07 *$ \\
\hline 3. Education ${ }^{1}$ & $4.11(1.32)$ & -.07 & $.21 * *$ & & $-.09 * *$ & $-.10^{* *}$ & $.10 * *$ & $.15^{* *}$ & $-.08 * *$ & $.22 * *$ & $.08^{*}$ & $-.15 * *$ & $.15^{* *}$ & .01 \\
\hline 4. Conservative Political Values ${ }^{2}$ & $2.29(0.89)$ & $.14 * *$ & -.06 & $-.10 *$ & & .03 & $-.11 * *$ & $-.22 * *$ & $.08 * *$ & $-.17 * *$ & -.05 & $.24 * *$ & $-.08^{*}$ & $-.12 * *$ \\
\hline 5. Trait Anxiety & $4.18(1.41)$ & $-.17 * *$ & $-.10^{*}$ & -.09 & .04 & & $-.14 * *$ & -.07 & $.09^{*}$ & -.08 & $.30 * *$ & .08 & $-.14 * *$ & $.14 * *$ \\
\hline 6. Cognitive Reflection & $0.39(0.40)$ & $.24 * *$ & $.12 *$ & $.12 *$ & $-.16^{* *}$ & $-.19 * *$ & & $.22 * *$ & $-.18^{* *}$ & $.20 * *$ & -.07 & $-.31 * *$ & $.28 * *$ & -.03 \\
\hline 7. AOT & $4.12(0.42)$ & $.11 *$ & .02 & $.18^{* *}$ & $-.29 * *$ & -.06 & $.35 * *$ & & $-.13^{* *}$ & $.27 * *$ & .03 & $-.31 * *$ & $.14^{* *}$ & $.14 * *$ \\
\hline 8. Faith in Intuition & $3.42(0.77)$ & -.06 & .02 & $-.09 *$ & .07 & $.12 *$ & $-.22 * *$ & $-.16^{*}$ & & $-.09 *$ & .04 & $.28 * *$ & $-.16^{* *}$ & .05 \\
\hline 9. Science Curiosity & $3.04(0.60)$ & .07 & -.02 & $.23 * *$ & $-.21 * *$ & $-.10^{*}$ & $.29 * *$ & $.37 * *$ & $-.11 *$ & & .03 & $-.20 * *$ & $.11 *$ & $.19^{* * *}$ \\
\hline 10. COVID-19 Worry & $5.26(1.33)$ & $-.17 * *$ & $.18^{*}$ & .08 & -.06 & $.32 * *$ & -.09 & .05 & .06 & .01 & & -.03 & -.05 & $.30 *$ \\
\hline 11. COVID-19 Unfounded Beliefs & $2.16(0.84)$ & $-.11 *$ & .06 & $-.17 * *$ & $.31 * *$ & .07 & $-.48 * *$ & $-.49 * *$ & $.34 * *$ & $-.28 * *$ & -.04 & & $-.29 * *$ & $-.13 * *$ \\
\hline 12. COVID-19 Knowledge & $0.77(0.17)$ & $.15^{* *}$ & .02 & $.15^{* *}$ & -.08 & $-.15^{* *}$ & $.34 * *$ & $.16^{* *}$ & $-.18^{* *}$ & $.15 * *$ & -.04 & $-.36^{* *}$ & & -.06 \\
\hline 13. COVID-19 Behavior & $4.39(0.53)$ & $-.28 * *$ & $.11 *$ & .04 & $-.17 * *$ & $.23 * *$ & -.02 & $.20 * *$ & .09 & $.23 * *$ & $.41 * *$ & $-.21 * *$ & -.01 & \\
\hline
\end{tabular}

AOT Active Open-minded Thinking

${ }^{1} 1$ = elementary school, $2=$ high school, 3 = bachelor's degree, $4=$ college, $5=$ graduate school, $6=\mathrm{PhD} ;{ }^{2} 1=$ extremely left/liberal, $3=$ center, $5=$ extremely right/conservative; $* p<.01, * * p<.001$

related worry. The indirect effect of anxiety on more responsible behavior via worry was significant $(0.061,95 \%$ CI $[0.040,0.092])$, indicating that the effect of trait anxiety on responsible behavior regarding $\mathrm{C}-19$ was fully mediated through C-19-related worry.

We explained $39.7 \%$ of variance of C-19-related unfounded beliefs. The beliefs were negatively predicted by cognitive reflection and actively open-minded thinking and positively predicted by faith in intuition, while the relationships of science curiosity and anxiety with the unfounded beliefs were non-significant. Unfounded beliefs significantly predicted less responsible behavior and the effects of cognitive reflection $(0.136,95 \% \mathrm{CI}[0.043,0.282])$, actively open-minded thinking $(0.111,95 \%$ CI $[0.035,0.262])$ and faith in intuition $(-0.037,95 \%$ CI $[-0.080,-0.012])$ on behavior were all mediated by unfounded beliefs. This suggested that individuals

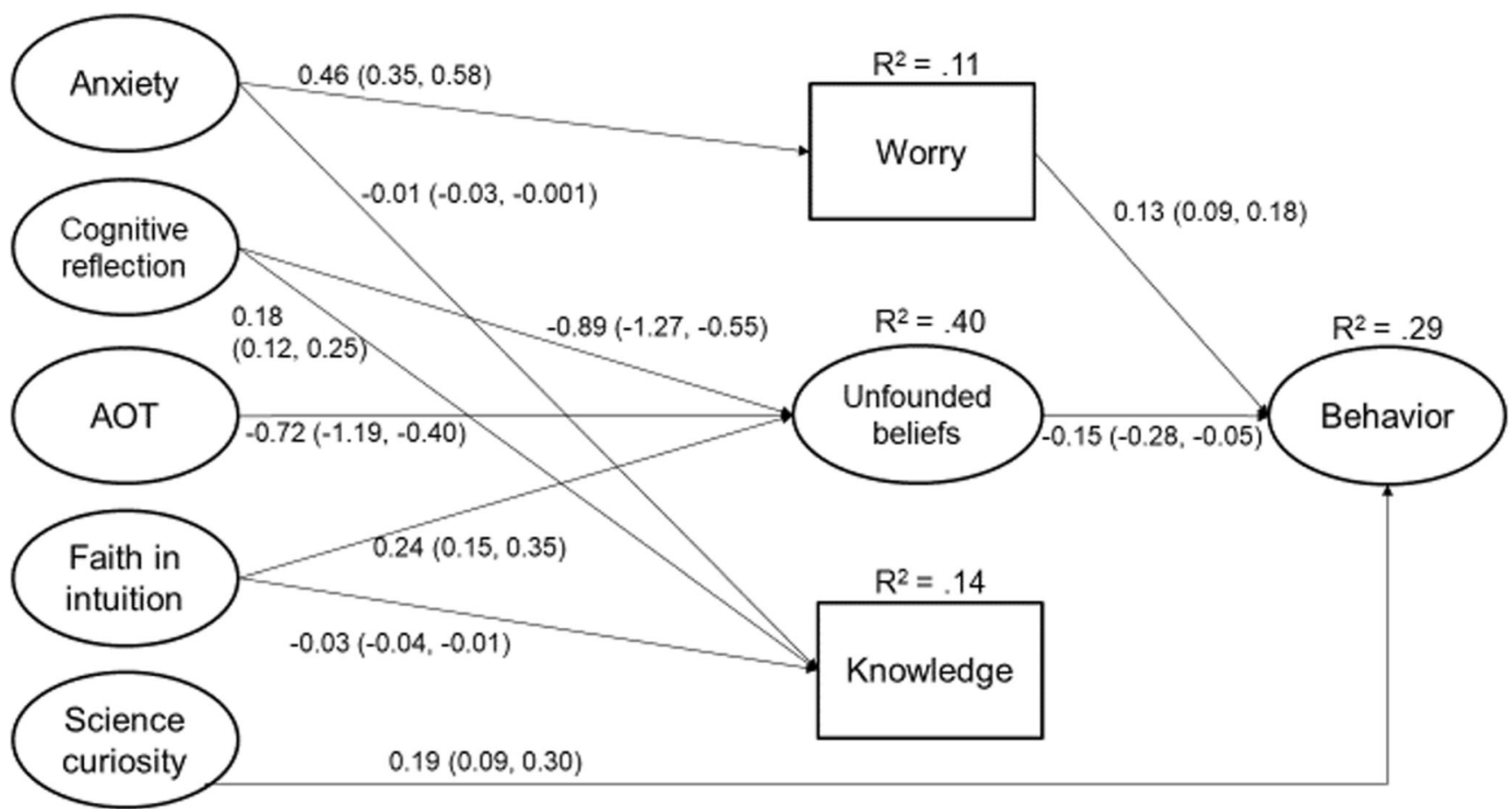

Fig. 1 Structural equation model for prediction of responsible behavior regarding COVID-19. Values shown are unstandardized regression coefficients and $95 \%$ confidence intervals calculated on 5000 bootstrap samples are in the parentheses. Latent variables are denoted as ellipse nodes and observed variables are denoted as rectangle nodes. AOT $=$ Actively open-minded thinking. $(n=962)$ 
who are less reflective, less open-minded and show more faith in intuition engage in less responsible C-19-related behavior due to the amount of unfounded beliefs they had about C-19.

The predictors from our model explained $13.5 \%$ of variance of C-19 related knowledge. Significant predictors were cognitive reflection (positive) and faith in intuition and trait anxiety (both negative), although the relationships with the latter two variables were very weak. Knowledge about C-19 did not predict $\mathrm{C}-19$ related responsible behavior.

Finally, once the other predictors in the model were controlled for, among individual difference variables only science curiosity remained a direct predictor of responsible behavior. In total, with our model we explained $28.6 \%$ of variance in C19 responsible behavior.

\section{Discussion}

Our main finding was that $\mathrm{C}-19$ responsible behavior follows both from emotions and cognitions about C-19 situation. Our correlation and SEM analyses showed that greater worry and weaker endorsement of C-19 unfounded beliefs lead to more responsible C-19 behavior. At the same time, knowledge of the most important facts about C-19 situation showed nonsignificant relationship to $\mathrm{C}-19$ behavior, though it correlated moderately positively with cognitive reflection and moderately negatively with C-19 unfounded beliefs.

We confirmed our expectation that trait anxiety would be a significant predictor of perceived threat related to the situation, and, in turn, lead to responsible C-19 behavior. In other words, dispositionally anxious individuals reacted to the current situation with more fear and worry and adhered to the recommendations about how to behave. Anxiety and worry had, as Sweeny and Dooley (2017) put it, an upside of making people behave in a more responsible way.

The unfounded beliefs had a significant negative effect on $\mathrm{C}-19$ responsible behavior: individuals that believe in conspiracy and unscientific claims about C-19 behave less responsibly (see Pavela Banai et al. 2020 for similar findings), supporting the idea of consequentiality of unfounded beliefs (Van Prooijen and Douglas 2018). Almost all explored psychological determinants were significant predictors of unfounded beliefs in the expected direction. Cognitive reflection and actively open-minded thinking predicted less and faith in intuition more endorsement of unfounded beliefs. Stated simply, less smart/rational individuals (cognitive reflection), that are reluctant to seek contrary evidence before making judgment (actively open-minded thinking) and trust their "gut feelings" (faith in intuition) more frequently endorsed unfounded C-19 beliefs. The three traits indirectly influenced responsible behavior through the unfounded beliefs.

It has to be noted that, even though in the model neither of these traits predicted behavior directly but through unfounded beliefs, unlike cognitive reflection and faith in intuition, actively open-minded thinking was correlated positively and significantly with responsible behavior. This is in line with some previous findings that actively open-minded thinking correlates with various outcomes even when other thinking dispositions do not. For example, actively open-minded thinking correlates with beliefs about global warming, vaccineautism relationship, evolution or stem cells research substantially higher than cognitive reflection (Pennycook et al. in press). However, actively open-minded thinking is not a unidimensional measure. Thus, in our study, we had omitted two items from the original actively open-minded scale due to their low correlation with the other items of the scale (see the method section). In addition to increasing internal consistency of the scale, this change also resulted in a stronger correlation with the criterion variable in comparison with the full scale. By looking at the content of the removed items, it seems that they are mostly related to one of the two major components of actively open-minded thinking, absence of unjustified high confidence. The remaining items are mostly related to the other component, absence of myside bias. Therefore, our results indicate that the tendency to question one's initial beliefs and look for counterarguments is at the core of AOT being a determinant of C-19 misbehaving.

While supporting consequentiality of unfounded beliefs, our data somewhat surprisingly revealed non-significant relationship between C-19 knowledge and responsible C-19 behavior. On the one hand, the result is surprising because one would expect that people who know more about the disease and its perils would be more careful in their behavior. On the other hand, the result is not so surprising given the extant literature on the "knowledge-behavior gap" showing that the relationship between knowledge and behavior is generally very small or moderate at best (Rimal 2000). Still, the question is why the beliefs are and the knowledge is not important determinant of $\mathrm{C}-19$ behavior? There are two possibilities. The first one probably has to do with the difference in conceptual proximity between the pairs of constructs. The beliefs are closer to the behavior than the knowledge in several ways. First, if one thinks that the whole C-19 narrative is a hoax or that the disease can be easily cured, one will be less motivated to behave in responsible ways. Second, if one has incorrect beliefs about the origin of the virus and ways of treating the disease, one will probably also have incorrect beliefs about the behavior. Finally, if one questions the official COVID-19 facts, one will also question official behavior recommendations. At the same time, the knowledge items dealt with broad concepts while behaviors were quite specific, and judging factual items to be true or false had less clear implications for the behavior (Ajzen et al. 2011). The second possibility relates to some deficiency of our knowledge measure. For example, one could question content validity of the measure. Namely, we asked our participants about the symptoms of the 
disease, high-risk groups, mortality rate, effectiveness of the vaccine and contagiousness of the virus. It can be argued that the knowledge of these facts is not relevant for behavior (i.e. whether one stays at home and avoids physical contacts, washes hands or sneezes in a handkerchief) and that we missed some more relevant aspects of the knowledge for this kind of behavior (i.e., knowledge about means through which the virus is transmitted). Our reasoning was that the knowledge measure should approximate one's general interest/ knowledgeability about the topic so that knowing more about one aspect of the virus will be related with knowing more about the other aspects. Although we still believe in the reasoning behind this approach, in the current research this was not the case. Therefore, the relationships among different aspects of knowledge and its relationship with behavior should be tested in future studies.

Contrary to our prediction, science curiosity did not predict endorsement of C-19 unfounded beliefs but had a substantial direct effect on responsible behavior. We can only speculate about the reasons here. Science curiosity might exhibit its influence on behavior not thorough its effect on unfounded beliefs but on some other variable that we did not capture with our model (e.g., trust in scientists or some relevant aspects of knowledge that we failed to assess).

In our study we failed to support efficacy of the "consider counter-arguments" technique on C-19 unfounded beliefs. Regardless of whether they were exposed to "consider counter-arguments" intervention or not, participants showed same levels of endorsement of C-19 related unfounded beliefs. We attribute this finding to the fact that our participants probably held a deeply developed beliefs about coronavirus/C-19 issues because they have been by far the most salient topics discussed in public sphere including news media, social networks and informal social contacts for weeks. Possibly people already came with firmly formed opinions and our simple intervention could do a little to change their cognitions.

Still, we believe that this does not mean that interventions aimed at inducing more actively open-minded way of thinking are useless in this situation. We partly derive this conclusion from our own data. In our model, actively open-minded thinking was a substantial predictor of lesser endorsement of C-19 unfounded beliefs and had significant indirect influence on responsible behavior. Therefore, it seems that teaching actively open-minded thinking could be beneficial, although these educational efforts should probably be more long-term oriented than a "one-shot" intervention (Baron et al. 2017).

Our findings are qualified by three limitations: we used one-point cross-sectional study, several of our measures were developed ad hoc for this study and our sample was selfselected resulting in our participants being mostly welleducated and probably more interested and knowledgeable about the topic than the general population would be. The last two facts probably restricted the range of responses on many of our variables. For example, more educated individuals probably scored higher that the general population would on actively open-minded thinking, cognitive reflection and COVID-19 knowledge while participants that decided to participate in our study were probably also more motivated for learning about the virus and possibly even more worried and anxious about the disease. The possible result of this range restriction due to self-selection effects in our sample is underestimation of the effects. In other words, we would expect the obtained effects to be higher in the general population than in our sample. In our view, the first limitation is, thus, more serious for the study's contributions. Due to our study design we cannot be in any way certain about the causal order among the constructs in our research model. However, we based the directions of the effects in our model on the theoretically grounded and empirically confirmed relationships between these variables and we believe that because of the urging need to confront the public health crisis, even this imperfect attempt could be informative to the interested audience.

\section{Conclusion}

Responsible C-19 behaviors follow from both perceived psychological threat related to corona virus and a more rational grasp of the current situation. Irresponsible behavior seems to be grounded in unfounded beliefs, and independent of the basic knowledge about the situation. In order to ensure adherence to guidance, responsible institutions should stress the perils of the situation and try to publicly scrutinize the most frequent unfounded beliefs about the corona virus and its effects. If the current situation persists in the medium term as expected (i.e., until the vaccine is found), psychologists could also encourage actively open-minded thinking in order to improve beliefs, judgments and behaviors related to the $\mathrm{C}-19$ crisis.

\section{Compliance with Ethical Standards}

Conflict of Interest On behalf of all authors, the corresponding author states that there is no conflict of interest.

Ethical Statement This research was approved by the Faculty of humanities and social sciences ethical committee.

Informed Consent With this research, we want to understand your thoughts on the coronavirus and the situation we are currently in. We are interested in your opinions about the coronavirus, the ways in which we can fight it, your behavior in the new situation and some psychological factors that could affect your thoughts and behaviors. The purpose of this research is to better understand our psychological adjustment to this crisis so that we can better deal with other, similar challenges in the future. You will not be exposed to any risks, stress or discomfort in this research. You will participate in it as an anonymous participant which guarantees the confidentiality of your identity. It will take you between 15 and $25 \mathrm{~min}$ to complete this questionnaire, depending on your personal pace of solving. We emphasize that participation in this research is voluntary 
and that you have the right to withdraw from it at any time without any consequences. If you have any complaints about the procedure, please contact etikapsi@ffzg.hr.

\section{References}

Aarnio, K., \& Lindeman, M. (2005). Paranormal beliefs, education, and thinking styles. Personality and Individual Differences, 39(7), 1227-1236. https://doi.org/10.1016/j.paid.2005.04.009.

Ajzen, I., Joyce, N., Sheikh, S., \& Cote, N. G. (2011). Knowledge and the prediction of behavior: The role of information accuracy in the theory of planned behavior. Basic and Applied Social Psychology, 33(2), 101-117. https://doi.org/10.1080/01973533.2011.568834.

Baron, J. (2018). Individual mental abilities vs. the world's problems. Journal of Intelligence, 6(2), 23. https://doi.org/10.3390/ jintelligence6020023.

Baron, J. (2019). Actively open-minded thinking in politics. Cognition, 188, 8-18. https://doi.org/10.1016/j.cognition.2018.10.004.

Baron, J., Scott, S., Fincher, K., \& Metz, S. E. (2015). Why does the cognitive reflection test (sometimes) predict utilitarian moral judgment (and other things)? Journal of Applied Research in Memory and Cognition, 4(3), 265-284. https://doi.org/10.1016/j.jarmac. 2014.09.003.

Baron, J., Gürçay, B., \& Metz, S. E. (2017). Reflection, intuition, and actively open-minded thinking. In M. Toplak \& J. Weller (Eds.), Individual differences in judgment and decision making: A developmental perspective. London: Psychology Press.

Burke, B. L., Kosloff, S., \& Landau, M. J. (2013). Death goes to the polls: A meta-analysis of mortality salience effects on political attitudes. Political Psychology, 34(2), 183-200. https://doi.org/10.1111/pops. 12005.

Erceg, N., Galić, Z., \& Bubić, A. (2019). "Dysrationalia" among University students: The role of cognitive abilities, different aspects of rational thought and self-control in explaining Epistemically suspect beliefs. Europe's Journal of Psychology, 15(1), 159-175. https://doi.org/10.5964/ejop.v15i1.1696.

Erceg, N., Galić, Z., \& Ružojčić, M. (2020). A reflection on cognitive reflection - Testing convergent validity of two versions of the cognitive reflection test. https://doi.org/10.31234/osf.io/ewrtq.

Frederick, S. (2005). Cognitive reflection and decision making. Journal of Economic Perspectives, 19(4), 25-42. https://doi.org/10.1257/ 089533005775196732

Hartley, C. A., \& Phelps, E. A. (2012). Anxiety and decision-making. Biological Psychiatry, 72(2), 113-118. https://doi.org/10.1016/j. biopsych.2011.12.027.

Imhoff, R., \& Bruder, M. (2014). Speaking (un-) truth to power: Conspiracy mentality as a generalised political attitude. European Journal of Personality, 28(1), 25-43. https://doi.org/10.1002/per. 1930.

Johnson, J. A. (2014). Measuring thirty facets of the five factor model with a 120-item public domain inventory: Development of the IPIPNEO-120. Journal of Research in Personality, 51, 78-89. https:// doi.org/10.1016/j.jrp.2014.05.003.

Kahan, D. M., Landrum, A., Carpenter, K., Helft, L., \& Hall Jamieson, K. (2017). Science curiosity and political information processing. Political Psychology, 38, 179-199. https://doi.org/10.1111/pops. 12396.

Lambert, A. J., Schott, J. P., \& Scherer, L. (2011). Threat, politics, and attitudes: Toward a greater understanding of rally-'round-the-flag effects. Current Directions in Psychological Science, 20(6), 343348.

Lilienfeld, S. O., Ammirati, R., \& Landfield, K. (2009). Giving debiasing away: Can psychological research on correcting cognitive errors promote human welfare? Perspectives on Psychological Science, 4(4), 390-398. https://doi.org/10.1111/j.1745-6924.2009.01144.x.

McCaul, K. D., \& Mullens, A. B. (2003). Affect, thought, and selfprotective health behavior: The case of worry and cancer screening. Social Psychological Foundations of Health and Illness, 1, 137168. https://doi.org/10.1002/9780470753552.ch6.

McPhetres, J., \& Pennycook, G. (2019). Science beliefs, political ideology, and cognitive sophistication. https://doi.org/10.31219/osf.io/ ad9v7.

Morewedge, C. K., Yoon, H., Scopelliti, I., Symborski, C. W., Korris, J. H., \& Kassam, K. S. (2015). Debiasing decisions: Improved decision making with a single training intervention. Policy Insights From the Behavioral and Brain Sciences, 2(1), 129-140. https:// doi.org/10.1177/2372732215600886.

Norris, P., Pacini, R., \& Epstein, S. (1998). The rational-experiential inventory, short form. Unpublished inventory. University of Massachusetts at Amherst.

Pavela Banai, I., Banai, B., \& Mikloušić, I. (2020). Beliefs in COVID-19 conspiracy theories predict lower level of compliance with the preventive measures both directly and indirectly by lowering trust in government medical officials. https://doi.org/10.31234/osf.io/ yevq7.

Pennycook, G., Cheyne, J. A., Seli, P., Koehler, D. J., \& Fugelsang, J. A. (2012). Analytic cognitive style predicts religious and paranormal belief. Cognition, 123(3), 335-346. https://doi.org/10.1016/j. cognition.2012.03.003.

Pennycook, G., Cheyne, J. A., Barr, N., Koehler, D. J., \& Fugelsang, J. A. (2014). Cognitive style and religiosity: The role of conflict detection. Memory \& Cognition, 42(1), 1-10. https://doi.org/10.3758/ s13421-013-0340-7.

Pennycook, G., Fugelsang, J. A., \& Koehler, D. J. (2015). Everyday consequences of analytic thinking. Current Directions in Psychological Science, 24(6), 425-432. https://doi.org/10.1177/ 0963721415604610.

Pennycook, G., Cheyne, J. A., Koehler, D. J., \& Fugelsang, J. A. (2016). Is the cognitive reflection test a measure of both reflection and intuition? Behavior Research Methods, 48(1), 341-348. https://doi.org/ 10.3758/s13428-015-0576-1.

Pennycook, G., McPhetres, J., Zhang, Y., \& Rand, D. (2020). Fighting COVID-19 misinformation on social media: Experimental evidence for a scalable accuracy nudge intervention. https://doi.org/10.31234/ osf.io/uhbk9.

Pennycook, G., Cheyne, J. A., Koehler, D., \& Fugelsang, J. A. (in press). On the belief that beliefs should change according to evidence: Implications for conspiratorial, moral, paranormal, political, religious, and science beliefs. Judgment and Decision Making, 15(4), 476-498. http://journal.sjdm.org/20/200414/jdm200414.html

Putnick, D. L., \& Bornstein, M. H. (2016). Measurement invariance conventions and reporting: The state of the art and future directions for psychological research. Developmental Review, 41, 71-90. https://doi.org/10.1016/j.dr.2016.06.004.

Raykov, T. (2001). Estimation of congeneric scale reliability using covariance structure analysis with nonlinear constraints. British Journal of Mathematical and Statistical Psychology, 54(2), 315323. https://doi.org/10.1348/000711001159582.

Rimal, R. N. (2000). Closing the knowledge-behavior gap in health promotion: The mediating role of self-efficacy. Health Communication, 12(3), 219-237. https://doi.org/10.1207/s15327027hc1203_01.

Sadler-Smith, E. (2011). The intuitive style: Relationships with local/ global and verbal/visual styles, gender, and superstitious reasoning. Learning and Individual Differences, 21(3), 263-270. https://doi. org/10.1016/j.lindif.2010.11.013.

Sorokowski, P., Groyecka, A., Kowal, M., Sorokowska, A., Białek, M., Lebuda, I., et al. (2020). Information about pandemic increases negative attitudes toward foreign groups: A case of COVID-19 outbreak. https://doi.org/10.31234/osf.io/j23vt. 
Spielberger, C. D. (2010). State-trait anxiety inventory. The Corsini Encyclopedia of Psychology, 1-1. https://doi.org/10.1002/ 9780470479216 .corpsy0943.

Šrol, J. (2020). Individual differences in epistemically suspect beliefs: The role of susceptibility to cognitive biases. https://doi.org/10. 31234/osf.io/4jcf7.

Ståhl, T., \& Van Prooijen, J. W. (2018). Epistemic rationality: Skepticism toward unfounded beliefs requires sufficient cognitive ability and motivation to be rational. Personality and Individual Differences, 122, 155-163. https://doi.org/10.1016/j.paid.2017.10.026.

Svedholm, A. M., \& Lindeman, M. (2013). The separate roles of the reflective mind and involuntary inhibitory control in gatekeeping paranormal beliefs and the underlying intuitive confusions. British Journal of Psychology, 104(3), 303-319. https://doi.org/10.1111/j. 2044-8295.2012.02118.x.

Svedholm-Häkkinen, A. M., \& Lindeman, M. (2018). Actively openminded thinking: Development of a shortened scale and disentangling attitudes towards knowledge and people. Thinking \& Reasoning, 24(1), 21-40. https://doi.org/10.1080/13546783.2017.1378723.

Swami, V., Voracek, M., Stieger, S., Tran, U. S., \& Furnham, A. (2014). Analytic thinking reduces belief in conspiracy theories. Cognition, 133(3), 572-585. https://doi.org/10.1016/j.cognition.2014.08.006.
Sweeny, K., \& Dooley, M. D. (2017). The surprising upsides of worry. Social and Personality Psychology Compass, 11(4), e12311. https:// doi.org/10.1111/spc3.12311.

Teovanovic, P., Lukic, P., Zupan, Z., Lazić, A., Ninković, M., \& Zezelj, I. (2020). Irrational beliefs differentially predict adherence to guidelines and pseudoscientific practices during the COVID-19 pandemic. https://doi.org/10.31234/osf.io/gefhn.

Van Prooijen, J. W. (2019). Belief in conspiracy theories: Gullibility or rational scepticism? In J. P. Forgas \& R. Baumeister (Eds.), The social psychology of gullibility: Conspiracy theories, fake news and irrational beliefs (pp. 319-333). New York: Routledge.

Van Prooijen, J. W., \& Douglas, K. M. (2017). Conspiracy theories as part of history: The role of societal crisis situations. Memory Studies, 10(3), 323-333. https://doi.org/10.1177/1750698017701615.

Van Prooijen, J. W., \& Douglas, K. M. (2018). Belief in conspiracy theories: Basic principles of an emerging research domain. European Journal of Social Psychology, 48(7), 897-908. https:// doi.org/10.1002/ejsp.2530.

Publisher's note Springer Nature remains neutral with regard to jurisdictional claims in published maps and institutional affiliations. 